Out of Equilibrium Dynamics

\title{
Ultra intense laser/plasma interaction at normal incidence: Relativistic mirrors effects, high harmonics generation and absorption
}

\author{
Javier Sanz $^{\mathrm{a}, *}$, Arnaud Debayle ${ }^{\mathrm{a}}$, K. Mima ${ }^{\mathrm{a}, \mathrm{b}}$ \\ ${ }^{a}$ ETSI Aeronáuticos, Universidad Politécnica de Madrid, 28040 Madrid, Spain \\ ${ }^{\mathrm{b}}$ School for the Creation of Photonic Industries, Shizuoka 431-1202, Japan
}

\begin{abstract}
An analytical study of the relativistic interaction of a linearly-polarized laser-field of $\omega$ frequency with highly overdense plasma is presented. Very intense high harmonics are generated produced by relativistic mirrors effects due to the relativistic electron plasma oscillation. Also, in agreement with 1D Particle-In-Cell Simulations (PICS), the model self-consistently explains the transition between the sheath inverse bremsstrahlung (SIB) absorption regime and the $\mathrm{J} \times \mathrm{B}$ heating (responsible for the $2 \omega$ electron bunches), as well as the mean electron energy.
\end{abstract}

Ultra intense ( $>10^{18} \mathrm{~W} \mathrm{~cm}^{-2}$ ) short laser pulse interaction with highly overdense plasmas offers very promising applications such as coherent and incoherent x-ray production, high harmonic generation, ion acceleration, and high-energy electron production [1,2]. When a relativistic intense laser pulse irradiates a solid density target it creating relativistic oscillating plasma mirror (ROM) by making the electrons oscillate around their rest positions. At such relativistic laser intensities, the dominant absorption mechanisms are collisionless [3]. However, even in this "simple" case of a laser pulse at normal incidence interacting with a steep plasma gradient, a plethora of absorption mechanisms exists in the literature: the ponderomotive $\vec{J} \times \vec{B}$ heating [4], different sort of skin effects [5], vacuum heating and many other mechanisms. Due to the complex mixing of all these processes, the basic physics is poorly understood. More recently, PIC codes have proven to be very powerful tools to study the laser/plasma interaction [6,7]. Their main limitation is the persistent difficulty to distinguish between cause and consequence. Moreover, some essential physical features about the electron distribution function (EDF) are not well captured, unlike a Vlasov description. To understand the basic physics of laser/overdense-plasma interaction precisely, it is necessary to revisit the electron dynamics on a thin layer of solid target surface, where electrons are accelerated. For relativistic laser intensities, and plasma density and temperature such that the initial relativistic electron pressure is negligible compared to the radiation pressure, electrons are pushed into the solid forming a very steep density profile. The relevant forces involved in the macroscopic motion of electrons are the ponderomotive and the longitudinal electric field caused by the strong electric charge separation effect. Hence a cold fluid approximation is expected to give us a reasonable physical description. This cold fluid approximation lies on the fact that the laser energy absorption $\left(\alpha_{T}\right)$ is small at normal incidence, and consequently the electron kinetic pressure $\sim$ (electron-energy-flux)/c $\sim \alpha T$ roughly, is also small. The 1D study we present combines the results of a cold fluid approximation, PICS [8], and the analysis of the EDF in a Vlasov description. The self-consistent description of the plasma surface oscillations allows us to determine the relativistic mirror equations (Eqs. (1), (2)), the laser-plasma fields scaling laws, and harmonic generation. Then, the mean electron energy and laser absorption are obtained and compared to PICS. The news finding of this work concern not only the self-consistent cold fluid approximation but also the numerical analysis carried out with PICS. Firstly, a transition regime for the high order

\footnotetext{
* Corresponding author.

E-mail address: franciscojavier.sanz@upm.es (J. Sanz).
} 
harmonic $(\nu \omega)$ generation (HHG) spectrum has been analytically derived. For laser intensities larger than a critical value $I_{c}\left(\mathrm{Wcm}^{-2}\right) \simeq 10^{-2} \times n_{0}\left(\mathrm{~cm}^{-3}\right) \times\left(\log \left(\omega_{p} / \sqrt{5} \omega\right)\right)^{2}$, where $n_{0}$ is the overdense density and $\omega_{p}$ is the plasma frequency, the reflected light spectrum $I_{v}$ decays as $\nu^{-5 / 2}$ in agreement with the analysis of Ref. [9], but for laser intensities less than $I_{C}$ the reflected light spectrum $I_{v}$ decays as $v^{-4}$ in agreement with our PIC simulations. Secondly, the numerical analysis we have performed of the EDF in a Vlasov description, using both the fields of the fluid model and PICS, shows the so-called chaotic scattering [10] of electron particles in momentum space, which explains partially the new results concerning the non-monotonic behavior of absorption with laser intensity. Thirdly, some physical arguments are given in order to explain the transition between the sheath inverse bremsstrahlung absorption regime [5] and the $\mathrm{J} \times \mathrm{B}$ heating, responsible for the $2 \omega$ electron bunches [4].

We consider a half-space $x \geqslant 0$ initially filled by neutral and homogeneous plasma of density $n_{0}$. Collisions and ion dynamics will be ignored. We assume a linearly polarized plane wave normally incident on the plasma for a long time. Let $e \vec{A} / m_{e} c \equiv a(x, t) \vec{e}_{y}$ and $e \Phi / m_{e} c^{2} \equiv \phi(x, t)$ be the dimensionless vector and electrostatic potentials, and $a_{0} \cos (\omega x / c-\omega t)$ the incident wave. Electron momentum and density, space and time coordinates are normalized such as: $\vec{p} \rightarrow \vec{p} / m_{e} c$, $n \rightarrow n / n_{c},(x, y) \rightarrow(x, y) \omega / c, t \rightarrow \omega t$, respectively. Here, $n_{c} \equiv \omega^{2} \varepsilon_{0} m_{e} / e^{2}$ is the critical density. The plasma is assumed to be highly overdense with a plasma frequency $\omega_{p}=\omega \sqrt{n_{0}} \gg \omega$. The magneto-electric fields are $B_{z}=a_{x}, E_{y}=-a_{t}$, and $E_{x}=-\phi_{x}$. Let $H \equiv \gamma-\phi$ be the Hamiltonian of an electron test particle with $\gamma \equiv \sqrt{1+p_{x}^{2}+\left(P_{y}+a\right)^{2}}$, and $P_{y}$ $\left(=p_{y}-a\right)$ the conserved canonical momentum. The EDF, $f\left(x, p_{x}, P_{y}, t\right)$, obeys the Liouville equation $\mathrm{d} f / \mathrm{d} t=0$, where $a, \phi$ are obtained from the wave $\left(a_{t t}-a_{x x}=j_{y}\right)$ and Poisson $\left(\phi_{x x}=n-n_{0} h(x)\right)$ equations. Here $h(x)$ is the step function and $j_{y} \equiv-\int\left(a+P_{y}\right) \gamma^{-1} f \mathrm{~d} p_{x} \mathrm{~d} P_{y}$. In a cold fluid approximation, the macroscopic equations for the electrons are the continuity and momentum along $x$. For relativistic laser intensities electrons are pushed into the solid forming a very steep density profile. For laser intensities such as the laser amplitude in the plasma surface $a_{s}\left(\sim a_{0} n_{0}^{-1 / 2}\right)$ is of order of unity, the electrons pushed inside the solid oscillate with $2 \omega$ frequency [11]. Let $\lambda_{s}(t)$ be the position of the electron plasma boundary (EPB), then $E_{x}=n_{0} x h(x)$ for $x \leqslant \lambda_{s}$. Let $x\left(x_{0}, t\right)$ be the trajectory of a fluid particle such that $x\left(x_{0}, t_{0}\right)=x_{0} \geqslant \lambda_{s}\left(t_{0}\right)$. Using Poisson and continuity equations, the momentum equation reads $\mathrm{d} p / \mathrm{d} t=n_{0}\left(x_{0} h\left(x_{0}\right)-x h(x)\right)+\phi_{x_{0}}-\gamma_{m}^{-1} a a_{x}$, where $p \equiv \gamma_{m} \dot{x}$ is the electron $x$-momentum, $\gamma_{m} \equiv \sqrt{1+a^{2}+p^{2}}$ is the relativistic factor and the dot means the time derivative. Taking $x_{0}=\lambda_{S}\left(t_{0}\right)$ we get the equation for $\lambda_{S}(t)$ :

$$
\frac{\mathrm{d}}{\mathrm{d} t}\left(\dot{\lambda}_{s} \sqrt{\frac{1+a_{s}^{2}}{1-\dot{\lambda}_{s}^{2}}}\right)+n_{0} \lambda_{s} h\left(\lambda_{s}\right)+a_{s} a_{x s} \sqrt{\frac{1-\dot{\lambda}_{s}^{2}}{1+a_{s}^{2}}}=0,
$$

where $a_{s} \equiv a\left(\lambda_{s}, t\right)$ and $a_{x s} \equiv a_{x}\left(\lambda_{s}, t\right)$. The second term is the electrostatic force ( $\sim \omega_{p}$ oscillations), and the right one the ponderomotive ( $\sim 2 \omega$ frequency). In $x<\lambda_{S}$ the EM wave contains the incident plus the reflected, $a_{r}(x+t)$, wave. The continuity of both $a$ and $a_{x}$ at $x=\lambda_{s}$, yields the equation governing $a_{s}(t)$,

$$
\dot{a}_{s}=\left(\dot{\lambda}_{s}+1\right) a_{x s}+2 a_{0} \sin \left(\lambda_{s}-t\right),
$$

and $a_{r}(\tau \equiv x+t)=a_{S}\left(t^{\prime}\right)-a_{0} \cos \left(\lambda_{S}\left(t^{\prime}\right)-t^{\prime}\right)$ with $t^{\prime}=\tau-\lambda_{S}\left(t^{\prime}\right)$. Thus $\lambda_{S}(t), a_{S}(t)$ are determined if $a_{x S}$ is known. The wave equation in $x>\lambda_{S}$ is solved in an approximate way (valid in $x-\lambda_{S} \sim n_{0}^{-1 / 2}$ ) taking the asymptotic leading order solution in an expansion in $n_{0} \gg 1$ with $a_{0} / \sqrt{n_{0}}=O(1)$, hence $\lambda_{s}=O\left(1 / \sqrt{n_{0}}\right)$ and the fluid electron $x$-momentum $p=O\left(1 / \sqrt{n_{0}}\right)$. Firstly notice that $\partial_{t} \sim O(1)$ while $\partial_{x} \sim O\left(\sqrt{n_{0}}\right)$ and consequently wave propagation effects $\left(a_{x x} \sim n_{0} a \gg a_{t t}\right)$ are neglected in the relevant layer. Secondly, in the expression for $j_{y} \equiv-n a / \gamma_{m}$, the fluid electron $x$-momentum is neglected in $\gamma_{m}=$ $\gamma_{0}\left(1+O\left(n_{0}^{-1}\right)\right)$, with $\gamma_{0} \equiv \sqrt{1+a^{2}}$, and finally using Poisson and momentum equation $n=n_{0} h(x)+\gamma_{0 x x}\left(1+O\left(n_{0}^{-1}\right)\right)$. Thus the simplified wave equation becomes $a_{x x} \simeq\left(n_{0} h(x)+\gamma_{0 x x}\right) a / \gamma_{0}$. Its solution in $x>0$ reads $a=2 \operatorname{sgn}\left(a_{*}\right) \theta \sqrt{\theta^{2}-1}$, where $a_{*} \equiv a\left(\lambda_{S} h\left(\lambda_{S}\right), t\right)$, and

$$
\theta \equiv \frac{\sqrt{2} \tanh \left(n_{0}^{1 / 2}\left(x-\lambda_{S} h\left(\lambda_{S}\right)\right)\right)+\sqrt{1+\gamma_{*}}}{\sqrt{2}+\sqrt{1+\gamma_{*}} \tanh \left(n_{0}^{1 / 2}\left(x-\lambda_{S} h\left(\lambda_{S}\right)\right)\right)},
$$

with $\gamma_{*} \equiv \sqrt{1+a_{*}^{2}}$. In $0>x>\lambda_{s}, a=\sinh \left(c_{1} x+\alpha_{0}\right) ; \alpha_{0}, c_{1}$ are obtained from the continuity of both $a, a_{x}$ at $x=0$. If

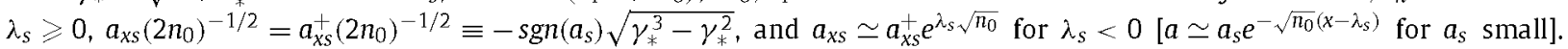
Then from (1), (2) it follows $\lambda_{s}(t), a_{s}(t)$. The solution establishes after few laser cycles, independently of initial conditions, toward an only one periodic solution: $a_{S}(t) 2 \pi$-periodic ( $\omega$ frequency), and $\lambda_{s} \pi$-periodic ( $2 \omega$ frequency) oscillating between $\lambda_{s \max }>0$ and $\lambda_{s \min }$. In Fig. 1a, we are plotting $\lambda_{s}(t) \sqrt{n_{0}}$ keeping $a_{0} n_{0}^{-1 / 2}=1$, and the equivalent PIC-EPB. Concerning PICS, the laser pulse is a 2 cycle linear ramp +11 cycles at constant intensity, with a plasma temperature of 500 eV. All comparisons are done during the thirteenth cycle. A weak but fast oscillation is superimposed $\left(\sim \omega_{p}\right)$ with the $2 \omega$ oscillations. This is a consequence of the electron-inertia together with the electrostatic force. This local fast oscillation becomes stronger when decreasing $n_{0}$. For $n_{0}$ fixed, the $2 \omega$ oscillation amplitudes increase with $a_{0}$, and above a critical value, $a_{0}>a_{0}^{c} \approx 1.5 \sqrt{n_{0}} \log \left(n_{0} / 5\right)$, according with our numerical study, the EPB remains inside the solid at any time. This trend has also been observed in our PICS for $a_{0}$ very large. The dynamics of the EPB is basically determined by the force balance between the electrostatic force given by $n_{0} \lambda_{s}$ and the ponderomotive force $a_{s} a_{x S} \sim a_{0}^{2} / \sqrt{n_{0}}$, therefore, the scaling 

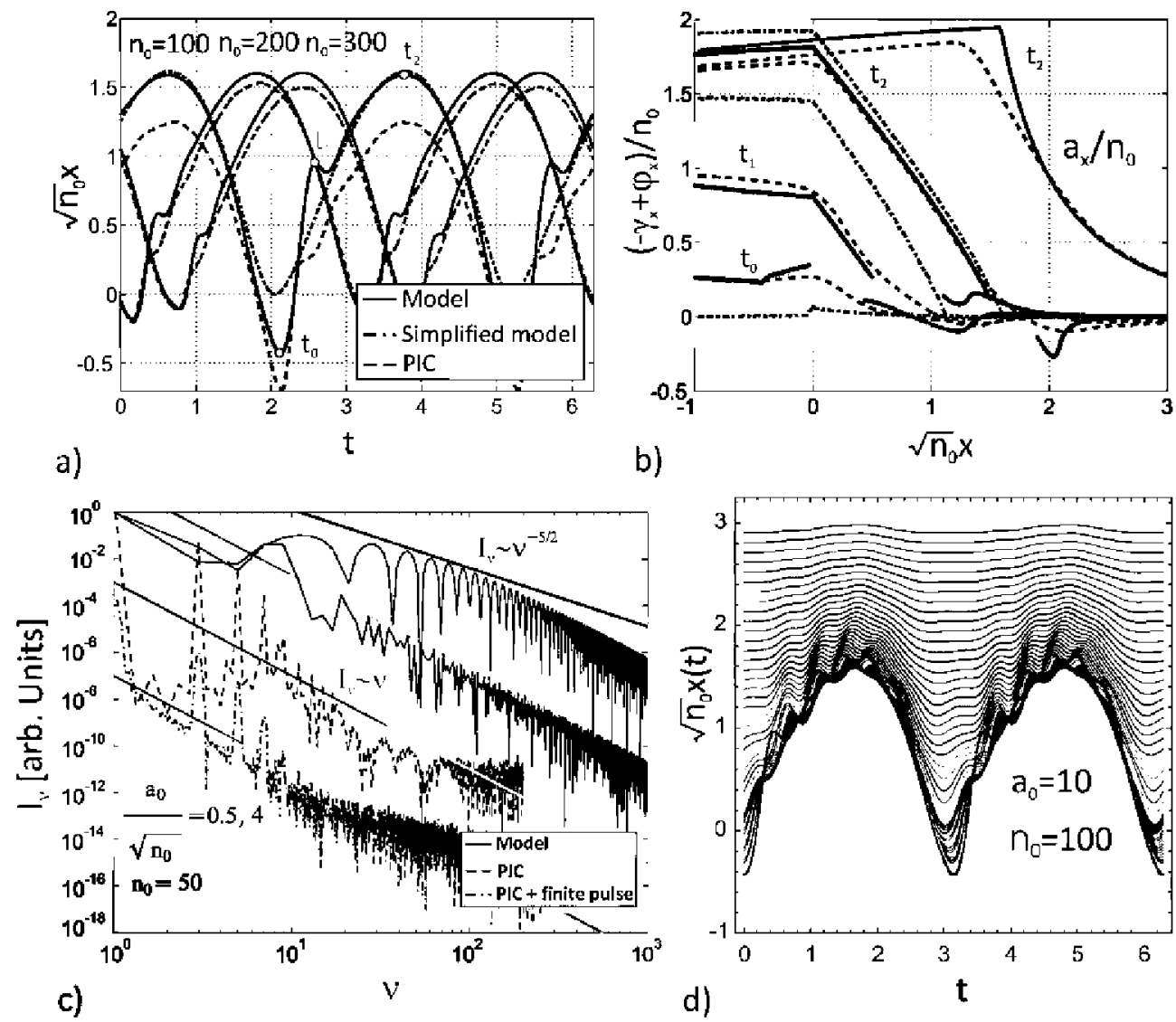

Fig. 1. a) Electron plasma boundary (EPB), versus time for 3 different densities $n_{0}$ and keeping $a_{0} / \sqrt{n_{0}}=1$ : model (solid lines), PICS (dashed lines) and simplified model (dashed-dotted lines). b) Total force, $-\gamma_{0 x}+\phi_{x}$, at different times (indicated in Fi. 3) obtained from the model (solid lines) and PICS (dashed lines). The dashed-dotted lines correspond to the simplified model. The magnetic field, $a_{x}$, obtained from the model (solid line) and PICS (dashed line) at the instant of time $t_{2}$ are also compared. All results were obtained for $a_{0}=10$ and $n_{0}=100 . \mathrm{c}$ ) High harmonic spectrum calculated from the model (solid lines) and from PICS (constant amplitude-dashed lines, finite pulse length-dashed-dotted lines). d) Electron fluid trajectories, $x\left(x_{0}, t\right)$, for $n_{0}=100$ and $a_{0}=10$. The EPB corresponds to the thick blue line.

on transition is mainly determined by the parameter $a_{0} / \sqrt{n_{0}}$. In the limit of $n_{0} \rightarrow \infty$ (keeping $\left.a_{0} n_{0}^{-1 / 2}=0(1)\right) n_{0}^{1 / 2} \lambda_{s} \geqslant 0$, and from (1), (2) it follows: $2 a_{0} \sin (t) \simeq a_{x s}^{+}, \lambda_{s} n_{0}^{1 / 2} \simeq\left|a_{s}\right| \sqrt{2 \gamma_{*}-2}$ (also plotted in Fig. 1a in dashed-dotted line).

It is well known [9] that plasma surface oscillations are efficient to convert the laser fundamental mode into high harmonics. ROM results from an oscillatory extension to Einstein's prediction for the frequency up-shift of light reflected off a perfect mirror moving stationary at relativistic velocities toward the source of light (relativistic Doppler effect): A pulse of duration $\Delta t$ at frequency $\omega$ is shift to a frequency $\omega^{\prime}=4 \gamma^{2} \omega$, with the Lorentz factor $\gamma \gg 1$. Since the number of cycles in an electromagnetic pulse is a Lorentz-invariant the pulse is also compressed and the resultant pulse duration is $\Delta t / 4 \gamma^{2}$. Hence in principle it should be possible to achieve substantial frequency upshifts and extremely short pulses by reflecting a high power laser pulse of a mirror with suitable properties. The difficulty is of course to create a reflectivity structure moving at a velocity close to the speed of light. In previous works [9], it has been shown that the relativistic Doppler effect induced by the relativistic plasma mirror converts the laser frequency into a high order harmonic $(\nu \omega)$ generation (HHG) spectrum decreasing as $1 / v^{5 / 2-8 / 3}$, hence suggesting that the detail of the plasma mirror oscillation is not important. However, the present cold fluid model provides a consistent calculation of the HHG spectrum. Indeed, the reflected electric field is $E_{y r}=a_{0} \sin \left(t^{\prime}-\lambda_{s}\left(t^{\prime}\right)\right)-a_{x s}\left(t^{\prime}\right)$, where $t^{\prime}$ is given implicitly as a function of $x+t, \lambda_{s}\left(t^{\prime}\right)+t^{\prime}=x+t$. The transition $a_{0} \sim a_{0}^{c}$ has a significant effect on the high order harmonic ( $\left.\nu \omega\right)$ generation (HHG) spectrum. For $a_{0}<a_{0}^{c}$, the EPB oscillates crossing periodically the solid boundary with $\dot{a}_{x s}(t)$ discontinuous, and according to the Fourier theory the spectrum decays as $v^{-4}$. But for $a_{0} \gg a_{0}^{c}$, the EPB reaches relativistic velocities and the maximum of $-\dot{\lambda}_{s}$ is almost the unity (light speed). Previous study [9] assumed that the laser electric field inside the plasma was negligible so that the reflected electric field is just $a_{0} \sin \left(t^{\prime}-\lambda_{S}\left(t^{\prime}\right)\right.$ ). But according with the cold fluid model the laser field amplitude inside the plasma is not negligible and the contribution of $a_{x s}\left(t^{\prime}\right)$ to the total laser electric field reflected is very important. As a consequence, the HHG spectrum, $I_{v} \sim\left(E_{y r}^{v}\right)^{2}$, decays approximately as $1 / v^{5 / 2-8 / 3}$ for $v$ less than a cutoff $v_{c}$ [9], but with very enhanced modes around the plasma frequency upshifted due to the Doppler effect. In Fig. 1c the HHG spectrum calculated with our model shows both types of ctrum decaying. The spectrum of 1 D PICS for both, constant pulse amplitude $\left(a_{0} n_{0}^{-1 / 2} \simeq 0.5\right.$, 
$n_{0}=50$ ), and finite pulse length with an exponential envelop of 10 laser cycles at $e^{-2}$, are also shown and decay as $v^{-4}$ as foreseen by the model.

One of the main concerns of the present work is the electron acceleration, responsible for laser absorption. Electrons are accelerated by the laser if they are driven towards the laser-plasma interaction region by the electrostatic field. The equation (above in the text) governing the particle path, $x\left(x_{0}, t\right)$, involves two eigenvalues, $\dot{x}_{0}, \phi_{x_{0}}$. They were obtained by imposing periodicity (see Fig. $1 \mathrm{~d})$. Then, $\phi_{x}=\phi_{x 0}+n_{0}\left(x_{0} h\left(x_{0}\right)-x h(x)\right)$. Plasma oscillations $\left(\sim \omega_{p}\right)$ beyond the EPB are clearly visible in Fig. 1d, and they are consequently present in the electrostatic field. The nearest trajectories to the EPB cross each other, hence resulting in the wave breaking (WB) phenomena in a layer of thickness $\Lambda_{W B} \sim \lambda_{S} / n_{0} \sim n_{0}^{-3 / 2}$, making the electrostatic field multivalued in such a layer. This effect is also illustrated in Fig. 1d. The WB, which appears in the exact resolution of the cold momentum equation, happens since there is no physical mechanism, like multi-stream flow or pressure ( $\omega_{p}$ dispersion), avoiding the crossing of one stream trajectories in the cold fluid electron momentum equation. In the limit of $n_{0} \rightarrow \infty$, WB disappears and $\phi_{x} \simeq \gamma_{0 x}$ inside the plasma. In Fig. 1b we are plotting the total force $\left(-\gamma_{0 x}+\phi_{x}\right)$ on an electron particle test $\left(p_{x}=0\right)$ at three instants of time, with $a_{0}=10, n_{0}=100$, and a good agreement with PICS is showed (except in the WB layers indicated in the figure). The WB will not be present in an asymptotic expansion for $n_{0} \gg 1$ of the electron momentum equation, as the electron inertia appears as a correction. We perform hence this approximation, to tackle the multi-valued electric field in the WB layer, by treating the electron fluid momentum, $p(x, t)$, as a perturbation $\left(O\left(n_{0}^{-1 / 2}\right)\right)$, which allows us to determine the longitudinal electric field by using $a(x, t)$ from Eq. (3). We take inside the plasma, from momentum equation, $\phi_{x} \simeq \gamma_{0 x}+p_{t}+\gamma_{0}^{-1} p\left(p-p \gamma_{0}^{-1} \gamma_{0 x}\right)$, where (from continuity equation) $p \simeq-n^{-1} \gamma_{0} \gamma_{0 x t}$. This field is showed in Fig. $1 \mathrm{~b}$ in dashed-dotted line. Then electrons mean energy and laser absorption are calculated in agreement with PICS.

Henceforth we focus on the kinetic description of electrons population and the laser absorption. Concerning this latter, general conservation laws derived from the Vlasov-Maxwell system, and time averaged (denoted by a bar), are useful to draw a picture of the whole mechanism. The total energy conservation (electrons and photons) yields $\overline{q_{h}}(\infty)=\alpha_{T} I_{0}$, where $q_{h} \equiv \int \gamma^{-1} p_{x}(\gamma-1) f \mathrm{~d} p_{x} \mathrm{~d} P_{y}$ is the electron kinetic energy flux, $I_{0} \equiv a_{0}^{2} / 2$ is the mean laser intensity and $\alpha_{T} \equiv$ $-I_{0}^{-1} \int \overline{j_{y} a_{t}} \mathrm{~d} x$ is the absorption coefficient. Moreover, defining the pressure $p_{h}$ as usual, electrons-photons momentum conservation reads $I_{0}\left(2-\alpha_{T}\right)=n_{0}(\bar{\phi}(0)-\bar{\phi}(\infty))+\bar{p}_{h}(\infty)$. Hence the pressure of photons, $I_{0}\left(2-\alpha_{T}\right)$, is balanced by the electrostatic force on the electrons and the kinetic pressure. In the cold fluid approximation, we obviously have $\bar{q}_{h}=$ $\alpha_{T}=0$. Our methodology to estimate the absorption is based on the calculation of the EDF evolution using the macroscopic fields of the cold fluid model and/or those obtained from PICS. Initially $(t=0)$, we assume an $\operatorname{EDF} f_{0} \sim \exp \left(-\left(p_{x 0}^{2}+\right.\right.$ $\left.p_{y_{0}}^{2}\right) / 2 T_{0}$ ), with temperature $T_{0}$ in $m_{e} c^{2}$ units $\left(\simeq 10^{-3}\right)$. We then apply the fields given by the simplified model, and after several laser cycles, we calculate the absorption from the electron energy flux. Also we have calculated the mean electron energy, $\overline{\gamma_{h}-1}$. Both, absorption and mean energies are also calculated from PICS, and showed in Fig. 2a. Concerning PICS, the absorption decreases with $a_{0}$ (regime A) [6] until a value of $a_{0} / n_{0} \sim 0.07$. Above such a value a strong increasing (regime B) of absorption, scaling as $\sim\left(a_{0} / n_{0}\right)^{3}$ according to PICS, is observed reaching the $20 \%$. Similarly, $\overline{\gamma_{h}-1}$ presents two tendencies. Our model, in solid line, reproduces these behaviors that can be justified by describing some features of the EDF near the EPB. A snap shot of such EDF for a PICS, is shown in Fig. 2b (regime B) at time $t=1(+12$ laser cycles). The total ponderomotive force is also plotted to show that the laser coupled with the electrostatic field acts as a "piston" on the plasma and generates the $2 \omega$ electron bunch. The electron energy gain of this latter can be deduced from Liouville's theorem. Phase space volume conservation allows us to get $f\left(p_{x}, P_{y}, x, t\right)=f_{0}$, with $P_{y}=p_{y 0}$ and $p_{x 0}=$ $p_{x 0}\left(p_{x}, p_{y 0}, x, t\right)$. The 4-variables function $p_{x 0}$ will provide essential qualities of the EDF. The $p_{x 0}$ function can be obtained integrating backward in time (BIT) the corresponding Hamiltonian system until $t=0$, or in the case of $2 \pi$-periodic regime, by integrating BIT until the out coming $\left(p_{x}<0\right)$ EDF is sited in an unperturbed region $x \gg n_{0}^{-1 / 2}$. The scattering function $p_{x 0}$ presents the features of the so-called chaotic scattering [10], with portions of non-regular behavior (chaotic and hence fractal) in $p_{x}, P_{y}$ and $t$. Such behavior appears as a consequence of the pseudo-trapping of electrons in phase space around the scattering region $\left(\sim n_{0}^{-1 / 2}\right)$. Notice that this stochastic feature differs from the stochastic motion of electrons captured in the laser standing wave, in the case of a pre-plasma with a scale length longer than the laser wavelength. Two examples of functions $p_{x 0}$ are presented in Fig. $2 c\left(P_{y} \equiv 0\right)$ in the case of PICS (regime B). The first one by integrating BIT from $t=1$, at the initial position indicated in dashed-dotted line in Fig. 1b. All thermal electrons are sited in the "pikes", below the graph $p_{x 0}=p_{x}$. These pikes correspond to the electron bunch crossing the dashed/dotted line in Fig. 2b. The same plot calculated from the simplified model is in good agreement concerning the electron bunch. The pike position, i.e. the electron momentum, depends on time since all electrons do not escape the laser at the same moment. This effect is seen with the $p_{x 0}$ calculated at a later time $(t \sim 1.2)$. The pike now contains more electrons with initially momentum $p_{x 0} \sim \sqrt{T_{0}}$. These latter leave the EPB region with at least the characteristic energy of an electron rebounding against the piston that scales as $E_{G} \sim \gamma \dot{\lambda}_{s} \sim a_{0}^{3} / n_{0}^{2}$. The number of scattered electrons every laser cycle is scaled by $n_{0} d_{s}$, with $d_{s}$ the equivalent width of the stochastic layer which is scaled as $\sim \lambda_{S} / n_{0}^{\mu}$, with $0<\mu<1$, roughly. Then the absorption efficiency $\alpha_{T} \sim$ $E_{G} \times n_{0} d_{s} / a_{0}^{2} \sim a_{0}^{3} / n_{0}^{\mu+5 / 2}$ (close to $a_{0}^{3} / n_{0}^{3}$ ). Our simplified model under-estimates the stochastic heating induced by the $2 \omega$ ponderomotive force coupled with the electrostatic oscillating field with plasma frequency, that can be seen in the PICS and in the full model but neglected in the simplified one (Fig. 1b). In the opposite extreme regime $A$, the penetration of the $\operatorname{EPB}\left(\sim a_{0}^{2} n_{0}^{-3 / 2}\right)$ is much smaller than the skin depth $\left(n_{0}^{-1 / 2}\right)$. The " $2 \omega$ electron bunch" or $\mathrm{J} \times \mathrm{B}$ heating, is thus negligible. 

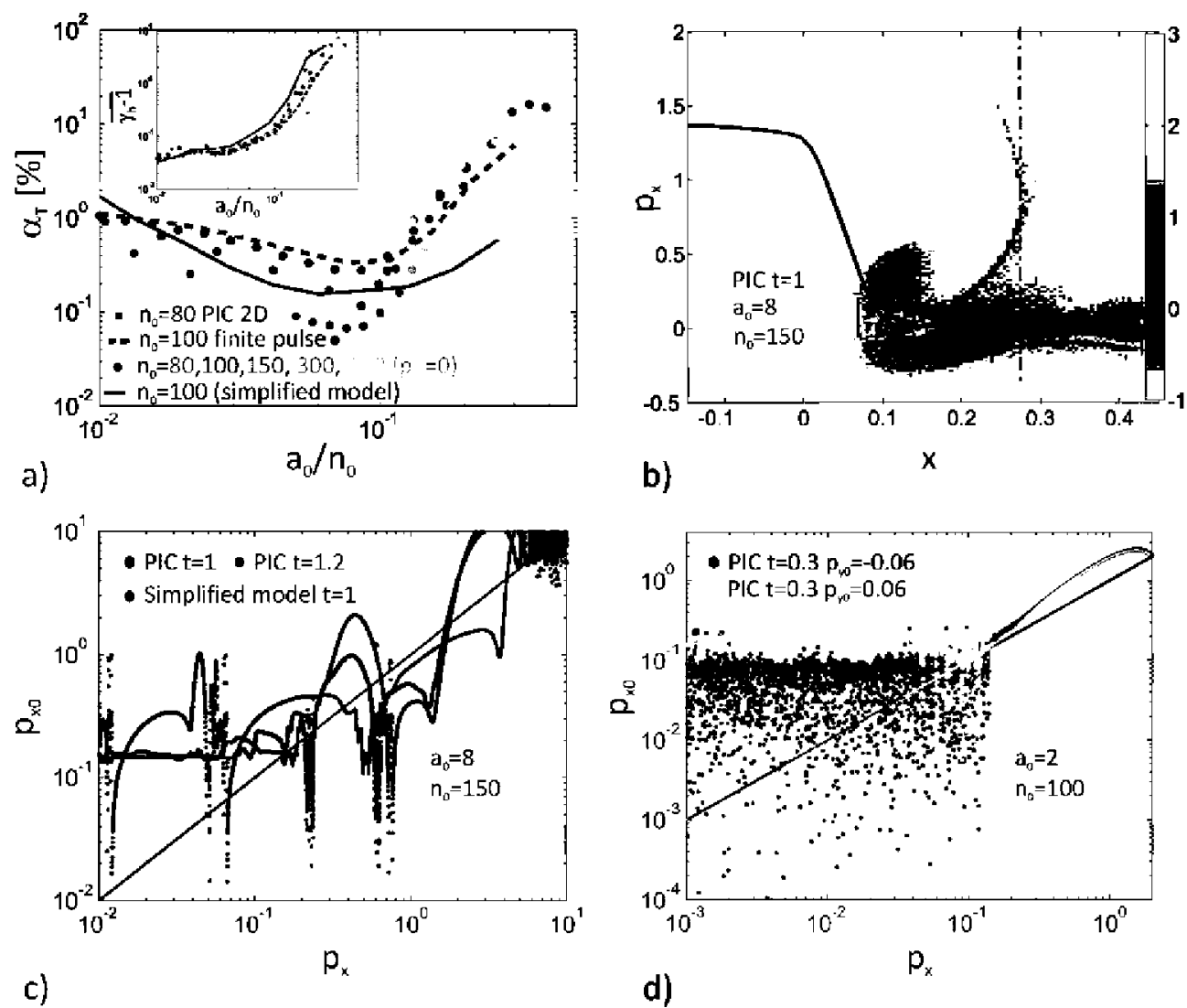

Fig. 2. a) Laser absorption and mean electron energy (inset) versus $a_{0} / n_{0}$, for several values of the initial overdense density $n_{0}$. Absorption and mean energy obtained from the simplified model (for $n_{0}=100$ ) are represented by the solid line. The figure also shows the results obtained from PICS: constant amplitude, finite pulse length and 2D PIC simulation. The absorption for particular case with $p_{y 0}=0$, is also represented in order to show that the transverse mechanical momentum does not play any role for absorption in regime $\mathrm{B}$. b) Phase space snap shot obtained from PICS at time $t=1(+24 \pi)$. The dashed-dotted vertical straight line is indicating the initial position of the electrons with momentum $p_{x}$, from where the Hamilton equations are integrated backward in time to determine $p_{x 0}$. It is also plotted the total ponderomotive force (solid line). c) Initial momentum $p_{x 0}$ against the final momentum $p_{x}$ obtained from PICS (for two different times) and the simplified model. Notice that the main contribution to the electron function distribution comes from the portions below the solid straight line $\left.p_{x}=p_{x 0} . \mathrm{d}\right)$ Initial momentum $p_{x 0}$ against the final momentum $p_{x}$ obtained from PICS for two values of the initial transverse momentum $p_{y 0}= \pm 0.06$, and $a_{0}=2, n_{0}=100$ (regime A).

In this latter case (close to a linear analysis), the main absorption mechanisms are the well-known SIB ( $n_{0} T_{0}<1$ ) or anomalous skin effect $\left(n_{0} T_{0}>1\right)$ [5], it is to say, the absorption comes from the imaginary part of the dielectric plasma function (see Eq. (42) in [5]), our results agree with that for $a_{0} / n_{0} \simeq 0.01$. Increasing $a_{0}$, in regime A, electrons can be trapped during many laser cycles before leaving the laser/plasma interaction region due to the electro-static field oscillation at the plasma frequency. This effect is illustrated in Fig. $2 \mathrm{~d}$, where the function $p_{x 0}$ presents a chaotic structure. The force applied to an electron is reduced to $\dot{p}_{x} \simeq-a_{x} p_{y 0} \gamma_{0}^{-1}$. Depending on the sign of $p_{y 0}$, the electron is repelled from the laser region or driven toward it. Again, this effect is illustrated in Fig. $2 \mathrm{~d}$ by the two plots of $p_{x 0}$ with $p_{y 0}$ of different signs. As seen before for $a_{0} / \sqrt{n_{0}}$ small, the absorption decreases with $a_{0}$. Such a decreasing is related with the decrement of the number of electrons going toward the laser interaction region. Notice that $\lambda_{S} \sim a_{0}^{2} / n_{0}^{3 / 2}$ is much less than the skin depth $\left(\sim 1 / \sqrt{n_{0}}\right)$ and the ponderomotive force, $\propto-\left(a+P_{y}\right) a_{x}$, push electrons with $\left|P_{y}\right|>p_{\min } \sim a_{0} / \sqrt{n_{0}}$ toward the laser in the skin depth region during one half-period, changing its sign in the next half-period. The number of $p_{x}$-electrons going toward the laser interaction region is $\sim \int_{p_{\min }}^{\infty} e^{-P_{y}^{2} / 2 T_{0}} \mathrm{~d} P_{y}$ and hence the decrement of electrons, and absorption, is proportional to $a_{0} / \sqrt{n_{0} T_{0}}$. To verify this assumption we have set $p_{y 0}=0$ in the PICS, and we observed the strong absorption decrease at low $a_{0} / n_{0}$ (blue symbols in Fig. 2a). 2D PICS, with the same finite pulse that for 1D PICS, and with an exponential profile with the HWHM radius $r_{0}=5 \lambda$ are presented in Fig. 2a. All tendencies are qualitatively reproduced. The differences concerning the absorption can be attributed to the coupling between the transverse electron momentum with the longitudinal one due to Weibel instability induced magnetic field [12].

In conclusion, we have developed a self-consistent model of laser/plasma interaction. The model foresees a nonmonotonic behavior of the laser absorption, and the high harmonic spectrum has been self-consistently derived. A transition regime for the high order harmonic generation spectrum has been analytically derived showing two types of spectrum decay in agreement with PIC simulations. 


\section{Acknowledgements}

This work was supported by the Ministerio de Ciencia e Innovación of Spain (Grant No. ENE2011-28489). The authors thank Y. Sentoku and E. d'Humières for sharing the PICLS code and L. Gremillet for the very prolific discussions.

\section{References}

[1] F.N. Beg, A.R. Bell, A.E. Dangor, C.N. Danson, A.P. Fews, M.E. Glinsky, B.A. Hammel, P. Lee, P.A. Norreys, M.a. Tatarakis, Phys. Plasmas 4 (1997) 447.

[2] T. Tanimoto, H. Habara, R. Kodama, M. Nakatsutsumi, K.A. Tanaka, K.L. Lancaster, J.S. Green, R.H.H. Scott, M. Sherlock, P.A. Norreys, R.G. Evans, M.G. Haines, S. Kar, M. Zepf, J. King, T. Ma, M.S. Wei, T. Yabuuchi, F.N. Beg, M.H. Key, P. Nilson, R.B. Stephens, H. Azechi, K. Nagai, T. Norimatsu, K. Takeda, J.R. Valente Davies, Phys. Plasmas 16 (2009) 062703.

[3] Y. Ping, R. Shepherd, B.F. Lasinski, M. Tabak, H. Chen, H.K. Chung, K.B. Fournier, S.B. Hansen, A. Kemp, D.A. Liedahl, K. Widmann, S.C. Wilks, W. Rozmus, M. Sherlock, Phys. Rev. Lett. 100 (2008) 085004.

[4] W.L. Kruer, Estabrook, Phys. Fluids 28 (1985) 430.

[5] T.Y. Brian Yang, William L. Kruer, Richard M. More, Bruce Landon, Phys. Plasmas 2 (1995) 3146.

[6] Erik Lefebvre, Guy Bonnaud, Phys. Rev. Lett. 74 (1995) 2002;

Erik Lefebvre, Guy Bonnaud, Phys. Rev. E 55 (1997) 1001.

[7] R. Mishra, Y. Sentoku, A.J. Kemp, Phys. Plasmas 16 (2009) 112704.

[8] Y. Sentoku, A. Kemp, J. Comput. Phys. 227 (2008) 6846.

[9] S. Gordienko, A. Pukhov, O. Shorokhov, T. Baeva, Phys. Rev. Lett. 93 (2004) 115002.

[10] Edward Ott, Tamás Tél, Chaos 3 (1993) 417.

[11] Wei Yu, M.Y. Yu, Z.M. Sheng, J. Zhang, Phys. Rev. E 58 (1998) 2456.

[12] Adam Héron, Laval, Phys. Rev. Lett. 97 (2006) 205006. 